Théologiques

Théologiques

\title{
Giulietta degli spiriti, une expérience post-moderne de possession
}

\section{Michel-M. Campbell}

Volume 5, numéro 1, mars 1997

Satan

URI : https://id.erudit.org/iderudit/024942ar

DOI : https://doi.org/10.7202/024942ar

Aller au sommaire du numéro

\section{Éditeur(s)}

Faculté de théologie de l'Université de Montréal

\section{ISSN}

1188-7109 (imprimé)

1492-1413 (numérique)

Découvrir la revue

Citer cet article

Campbell, M.-M. (1997). Giulietta degli spiriti, une expérience post-moderne de possession. Théologiques, 5(1), 42-58. https://doi.org/10.7202/024942ar
Résumé de l'article

Le Giulietta degli spiriti de Fellini, qui met en scène une femme des années 60, hantée, terrorisée par des présences, rappelle le genre des films de possession (par ex : les Exorcists). Une lecture psychologique réduit ces esprits à l'expression d'un complexe d'infériorité que l'héroïne parvient à dépasser. Cependant de nouveaux esprits bienveillants s'offrent à elle. Une lecture religiologique permet de voir ce film comme le voyage initiatique d'une post-moderne qui assume le rôle critique de l'imaginaire. Ce qui donne à penser à la théologie. 


\title{
Giulietta degli spiriti, une expérience post-moderne de possession
}

\author{
Michel-M. CAMPBELL \\ Faculté de théologie \\ Université de Montréal
}

\section{RÉSUMÉ}

Le Giulietta degli spiriti de Fellini, qui met en scène une femme des années 60 , hantée, terrorisée par des présences, rappelle le genre des films de possession (par ex : les Exorcists). Une lecture psychologique réduit ces esprits à l'expression d'un complexe d'infériorité que l'héroïne parvient à dépasser. Cependant de nouveaux esprits bienveillants s'offrent à elle. Une lecture religiologique permet de voir ce film comme le voyage initiatique d'une post-moderne qui assume le rôle critique de l'imaginaire. Ce qui donne à penser à la théologie.

F. Fellini's Giulietta degli spiriti, a depiction of a sixty-year-old woman haunted and terrorized by presences, recalls the genre of films of possession (e.g. The Exorcist). A psychological reading reduces these spirits to an expression of an inferiority complex, from which the heroine is reaching to surpass. However, new benevolant spirits offer themselves to her. A reliological reading allows a view of this film as the initiatory voyage of a postmodernist which assumes a critical role for the imagination. This gives food for thought for theology.

La commande d'un article sur Fellini, dans un numéro thématique sur le diable, m'a d'abord parue incongrue. Cependant son film Giulietta degli spiriti $^{1}$, reprend, à sa manière, le thème de la possession diabolique même 
s'il s'avère loin du genre de L'Exorciste ${ }^{2}$ où les personnages (entre autres, une jeune fille et un prêtre) se trouvent explicitement aux prises avec un démon. Les quelques références au clergé comme au diable, que l'on trouve dans Giulietta degli spiriti, présentent des figures tout au plus folkloriques $^{3}$. Pourtant ce film présente l'expérience d'une femme de notre temps, hantée par des figures lourdement chargées de connotations religieuses ou religiologiques. Je propose donc de « revisiter» ce film: tout d'abord de le revoir en le résumant; le relire comme une histoire de cas psychologique puis comme un voyage initiatique; pour, enfin, tirer quelques conclusions sur les figures du diable qu'on y trouve. Commençons donc par revoir ${ }^{4}$ ce film qui s'organise en une série discontinue de jours et de nuits que nous regrouperons en dix séquences ${ }^{5}$.

\section{Le film}

\section{Première soirée (sql)}

Dans la pinède d'Ostie, élégante plage/banlieue de Rome, une maison modèle. Giulietta y finit de s'habiller et de préparer un souper intime avec Giorgio, son mari. Celui-ci a oublié leur quinzième anniversaire de mariage même s'il arrive avec une kyrielle d'amis: Valentina, la fantaisiste, qui apporte à Giulietta un "chasse-esprits" de Los Angèles; Genius, le Maestro efféminé, spécialiste des sciences occultes; Cesarino, vieil avocat, amoureux transis de Giulietta; Dolorès, enfin, la sculptrice d'un âge certain et son gigolo, culturiste. Monde bourgeois d'artistes et de professionnels «libérés » des années 60 . Un couple cossu: le mari « fait dans les relations publiques "; la femme tient maison avec ses deux

2 Le film de Willam FREIDKIN, The Exorcist (ÉUA, 1973) a connu deux suites : le film de John BOORMAN, The Exorcist II : The Heretic, (ÉUA, 1977) et celui de William Peter BLATTY, The Exorcist III, (ÉUA, 1990).

3 On y trouve trois figures de prêtre : le premier, un évêque, est d'un conservatisme caricatural (sq3); le deuxième, un franciscain (religieux ou prêtre) s'avère plus ou moins imbécile (sq5); le troisième, le détective obséquieux est un laïc qui, à l'occasion, se travestit en "clergyyman" pour mener une enquête (sq4). La seule figure chnotienne est celle d'un gros jardinier (sq5) sympathique qui n'a rien de terrible (il a peur d'un chat).

4 L'accès à un film, même un classique, est beaucoup plus difficile qu'à un livre. Cela explique la longueur de notre résumé. Notons qu'il se limite à une translitération des actions et des dialogues et que nous ne tiendrons pas compte des éléments d'écriture proprement cinématographiques.

5 Nous identifierons chaque journée à une séquence (sq) numérotée (par ex: sq1), ce qui permettra les renvois à l'intérieur du texte. 
bonnes (Thérèse, bien en chair, qui a des rendez-vous galants et Elysabeth avec ses airs de "sainte nitouche ") et un jardinier.

Comme d'habitude, on se livre à une séance de spiritisme. Valentina demande à Iris, un esprit qui se présente, de "leur dire quelque chose de gentil qui les aide à vivre ». Le téléphone sonne mais c'est le silence au bout de la ligne. Olaf, un deuxième esprit, pour le moins impertinent, a un message pour Giulietta : «Elle n'est rien! Elle n'est personne! Elle ne compte pour personne! ». Giulietta s'évanouit tandis que le maestro proclame qu'elle est très douée (en italien, "dotata ") pour les esprits.

\section{Le lendemain ( $s q 2$ )}

Sur la plage, Giulietta, qui garde ses nièces jumelles, parle avec un ami médecin, de l'existence des esprits et de ses expériences d'enfants en ce domaine. Celui-ci ne croit pas aux esprits et, pour échapper à ce genre de fantasmes, il lui recommande de bien manger et de faire l'amour souvent. Giulietta ferme les yeux. Apparaît une barge sinistre, pleine de gens nus, d'allure barbare et misérable, qu'un vieillard tire péniblement. Giulietta prend sa relève avec peine. Le vrombrissement d'un avion la réveille. Arrive alors, dans une espèce de cortège solennel, Susie, sa riche voisine, fort sexy. Retour à la maison. Giulietta rencontre ses sœurs et sa mère dont l'élégance excentrique jure avec la simplicité de sa propre mise. Sa mère, d'une beauté impériale, refuse de l'embrasser parce que Giulietta serait en sueur. D'un air méprisant, elle lui recommande de soigner son apparence. Le soir, Giulietta tente de parler à Giorgio du retour de ses esprits mais il ne l'écoute pas. Durant la nuit, elle le surprend, dans son sommeil, à appeler tendrement une certaine Gabriella

\section{Troisième journée (sq3)}

Au déjeûner, Giorgio proteste: il ne connaît pas de Gabriella. Thérèsa, la bonne, se plaint : elle répond souvent au téléphone pour se buter sur un silence. Visite de Valentina. Celle-ci envie la facilité avec laquelle Giulietta assume son rôle de ménagère et avoue se sentir ellemême déracinée. Elle invite Giulietta à venir voir Bhisma, le fameux guru, hermaphrodite. Tonnerres et panne de courant les accueillent dans un grand hôtel romain. Impression de labyrinthe. Giulietta s'arrête devant une noce conventionnelle où un évêque ne trouve rien de mieux pour son discours que de répéter littéralement saint Paul. Les disciples de Bhisma se livrent à une série de paradoxes ésotériques : questions sur la réalité d'une pomme (apparence et forme) ou sur les portes de l'Enfer (la menace de la mort) et du Paradis (la levée de la menace). La séance se termine sur une faiblesse de Bhisma qui, malgré tout, accorde une audience privée à Giulietta. Dans une espèce de délire, il évoque le Kama Sutra: la 
" rencontre des corps comme combat », le sens des sons ( "put ", « quat", etc.) et "des morsures". "L'amour est une religion, ton mari est le dieu et toi, la prêtresse qui doit sacrifier sur l'autel de ton corps». Apparition de Susie, la voisine, en trapéziste. Giulietta, d'abord envoûtée par la transe de Bhisma, se rebiffe quand il dit que "l'amour est un métier». Elle fuit Bhisma qui parvient à lui annoncer un visiteur et évoquer le «breuvage de l'oubli, qui efface toute soif, même l'inavouable".

Retour en auto, dans l'orage. Giulietta se rappelle (flashback) la fuite de son grand-père - un enseignant de tendance laïque - avec une superbe trapéziste (qu'elle revoit, au cirque, sous l'apparence de Susie) malgré les protestations de son directeur d'école qui le fit alors interdire dans toutes les écoles du royaume. $\grave{A}$ son retour le grand-père fut aussi interdit chez sa propre fille, la mère de Giulietta, mais cette dernière, encore toute jeune, allait le voir en cachette.

À la maison, Giulietta trouve un invité de Giorgio, un espèce d'hidalgo, qui lui prépare une sangria en reprenant textuellement les termes de Bhisma ( "le breuvage... qui efface... la soif... inavouable "). Il fait l'éloge de la tauromachie: "le taureau meurt dans sa propre chimère... ce qui compte c'est la douceur des gestes, l'équilibre,... la pureté de la pensée ». Au lit, Giulietta voudrait échanger avec Giorgio qui proteste de sa fatigue. Durant la nuit, elle le surprend en train de téléphoner à sa maîtresse.

\section{Quatrième journée (sq4)}

Giulietta décide de faire surveiller son mari par une agence privée. Le détective (le vieil homme qui tirait la barge, sq2), plein de componction, s'assure qu'elle est bien décidée à tout savoir. Apparition du terrible directeur du grand-père, qui, comme un Maître de Justice, crie vengeance. Giulietta confirme sa demande. À son retour, elle évite le bel hidalgo et va chez Dolorès, qui lui fait part de sa théologie. Avant, Dolorès avait peur de Dieu; maintenant elle sait que Dieu, c'est un beau corps de mâle comme ceux qu'elle sculpte. Elle voudrait prendre Dieu comme amant. Giulietta confie, qu'enfant, elle croyait que Dieu se trouvait derrière une porte poussiéreuse.

Flashback. Une séance scolaire. Une série de religieuses, sans visage, toutes pareilles, mettent des ailes à de jeunes communiantes. Giulietta joue le rôle d'une martyre qui, ayant refusé d'abjurer, se retrouve en ascension vers le ciel sur un grill aux flammes de papier. Plus tôt, sa petite amie, Laura, l'a suppliée de parler d'elle à Dieu en arrivant là-haut. Giulietta se trouve suspendue, entre scène et plafond, devant une porte poussiéreuse quand, soudain, le spectacle est interrompu. Son scandaleux de grand-père, qui trouve cette scène barbare, vient la 
déliurer au grand dam de son directeur. "Et toi, dit-il, à Giulietta-enfant libérée, tu ne dis rien? Tu te laisses faire? »

\section{Autre journée (sq5)}

Giulietta rapporte son chat à Susie sa voisine. Luxueuse maison de style "modern art" mais dont les murs sont lézardés. Atmosphère ambigü. Jazz, sensualité (une voix dit à Giulietta qu'elle doit suivre les enseignements de Susie) avec, en mineur, des impressions de lupanar ou d'aile psychiatrique. Il y a là la vieille mère insomniaque de Susie, sa sœur suicidaire (comme Laura l'amie de Giulietta) et le fétichiste Aliocha. Susie semble heureuse dans la gloire de son corps de sex-symbol (dans sa chambre un lit rond sous un miroir; au pied du lit, la piscine où elle glisse après avoir fait l'amour). Cette euphorie est cependant souvent brisée par des scènes de colère de Susie qui ressemble alors plus à une tenancière qu'à une femme au faîte de sa gloire. Elle avoue à Giulietta qu'elle a déjà rêvé : Giulietta habillée en religieuse la grondait pour sa démarche lubrique. Susie avoue que souvent, en regardant par la fenêtre de sa chambre la maison de Giulietta, elle a envié son bonheur. Elle s'informe de sa vie: "Comment peux-tu aimer un seul homme?". Giulietta lui confie la vision qu'elle avait du mariage ("Il était tout pour elle et elle était toute pour lui ») mais Susie ne l'écoute pas et cherche à l'entraîner avec deux dragueurs. Giulietta s'excuse et rentre chez elle.

\section{Autre journée (sq6)}

Giulietta raconte à ses nièces l'histoire de "Sete d'un colpo ", qui n'a pas peur du diable et s'aventure dans le labyrinthe, quand elle est convoquée chez le détective. Celui-ci, toujours aussi obséquieux, cite Augustin ( la connaissance de l'autre est condition de l'amour véritable"). Il lui confirme la relation de Giorgio avec une Gabriella, mannequin.

\section{Le samedi soir chez Susie ( $s q 7$ )}

Un party du jet set, plein de références poétiques. On y joue à revivre une scène de bordel. Giulietta, qui est venue seule, demande à un mannequin si elle connaît une Gabriella et si celle-ci est plus belle qu'elle... et si elle n'est pas " un peu putain". "Ce qui, ajoute-t-elle, commence à devenir un compliment à mes yeux ». Long chant, plein de mélancolie. Giulietta est triste, comme Arlette. Arrive le filleul de Susie, éphèbe indien en pyjama blanc. Susie entraîne Giulietta vers sa chambre, car son filleul désire celle-ci. Tout est silence. Dans la nuit, Susie surprend des couples en train de faire l'amour. Giulietta se retrouve dans la chambre de Susie avec l'Indien langoureux. Toute la maisonné 
suspendue écoute ce qui va se passer. Une voix dit à Giulietta d'obéir à Susie mais, aussitôt, apparaît le grill sur lequelle brâle la martyre (qui, adulte, a le visage d'Elysabeth, la bonne masochiste). Giulietta s'enfuit.

\section{Le lendemain ( $s q 8)$}

Nouveau party, dans le jardin de Giulietta. Tous ses amis sont là et s'amusent à jouer au psychodrame avec une psychanalyste américaine. Dans la salle de bain, Giulietta, bourellée de remords au souvenir de la veille, promet (à Dieu?) de ne plus jamais recommencer. Les apparitions se multiplient (entre autres, celle d'une martyre adulte sur le grill qui s'avère nulle autre que Susie avec un air particulièrement lubrique). Giulietta, torturée, se joint à la fête. Elle n'écoute pas, se livre à un monologue intérieur: "Vous ne les voyez pas", crie-t-elle à ses invités en évoquant tous ses esprits, * je vis dans un monde où tout le monde parle, parle, me commande d'être belle, féminine, d'obéir! "Elle danse avec l'hidalgo. Elle est tellement désespérée qu'elle lui demande s'il est bien réel, s'il peut l'aider. "La vérité libère " lui répond-il sans se référer à Augustin et il se contente de lui dire, en citant un proverbe espagnol, qu'elle se suffit à elle-même, qu'il la souhaite heureuse. Giorgio disparaît. Césarino rappelle alors à Giulietta qu'il est maintenant plus facile d'obtenir un divorce pour adultère. "Peutil encore espèrer?" Départ des invités. Giulietta marche avec la psychanalyste. "Vous avez peur d'être abandonnée " conclut celle-ci. "Regardez les arbres qui croissent par eux-mêmes. Au fond, vous voulez de tout cœur que votre mari parte... mais vous avez peur d'être heureuse. »

\section{Une autre journée ( $s q 9)$}

Giulietta se rend chez Gabriella. Celle-ci n'y est pas. La bonne, qui prépare les bagages du mannequin, confie à Giulietta comment cette dernière semble heureuse avec son nouvel amant. Gabriella téléphone. Giulietta demande à lui parler pour s'entendre répondre froidement «qu'elles n'ont rien à se dire ". À la maison, Giorgio annonce un voyage à Milan, il avoue avoir eu une relation avec Gabriella, ("rien de grave $»)$, traverser une période d'incertitude et avoir besoin de temps pour réfléchir. Pour la première fois de leur vie de couple, il part sans que Giulietta ait préparé ses bagages.

Restée seule, Giulietta pleure. Laura, son amie, suicidée, lui offre de la suivre dans la mort (elle apparaît, dans sa robe de communiante, couchée sur le grill, mais sous l'eau). La maison est tour à tour envahie par l'hidalgo, le maestro, un couple de SS, le bel Indien. Puis ce sont Susie, le détective, Césarino, les soldats romains à cheval. En avion, le grand-père de Giulietta lui dit que, par sa faute à elle, il ne peut atterrir. Le directeur se dresse dans le hall, terrible avec une coiffure de feu. Giulietta supplie sa mère (plus belle et plus froide que 
jamais) de l'aider. Giulietta entend des pleurs. Une porte se dessine dans le mur. Giulietta s'en approche malgré les injonctions de sa mère : "Obéis à ta mère ". "Ne bouge pas! Tu ne dois pas! "Tu ne me fais plus peur», rétorque Giulietta. Aussitôt, la porte s'ouvre. Giulietta passe dans un recoin où elle se retrouve elle-même, enfant sur le fameux grill. La Giulietta-adulte s'efforce de dénouer les liens de la Giulietta-enfant. Elle y parvient. Alors sa mère et les autres esprits s'estompent.

\section{Le jour se lève (sq10)}

Son grand-père vient lui faire ses adieux. "Amène-moi avec toi! », supplie Giulietta. "Je ne suis qu'une de tes pensées " de répondre le grand-père, "mais toi, tu es la vie." Il s'envole avec Julie/Susie -la trapéziste toute souriante. Giulietta marche dans son jardin dont elle ouvre largement les portes. Il vente. Il fait bon. Giulietta est libérée. Des voix se font entendre. "Qui êtes-vous?", leur demande-t-elle. "Des amis », répondent les voix. Si tu nous veux, si tu nous veux, si tu nous veux, nous serons avec toi ». Giulietta marche calmement dans la pinède, accompagnée d'un air de trompette qui a la beauté du jour.

\section{Quelques pointes d'observation}

Avant d'entreprendre de relire ce film, on peut, d'entrée de jeu, souligner la présence de trois axes thématiques qui le traversent de part en part. Tout d'abord, celui de l'identité. Le thème de la gémelléité ou des doubles (similaires ou opposés), du miroir, se répète tout au long du film. Que l'on pense, entre autres, aux nièces jumelles identiques de Giulietta (sq2), à ses deux bonnes (sq1), à ses deux scurs qui ont hérité de l'élégance excentrique de leur mère contrairement à Giulietta (sq2), ou encore à Valentina et à Susie qui envient le bonheur de Giulietta (sq3 et sq5) alors que celle-ci est tentée de s'identifier à Susie (sq5). Lorsque la religieuse met la dernière main au costume de Giulietta-enfant (martyr), elle proclame : «tu es exactement comme la sainte»(sq5).

Un deuxième thème, omniprésent, est celui de la sexualité. L'infidélité de Giorgio, les déclarations intempestives de Dolorès, le monde de Susie renvoient la candide Giulietta à sa propre vie sexuelle: le médecin lui recommande de faire l'amour souvent $(\mathrm{sq} 2)$ et elle est elle-même tentée de suivre les pas de sa voisine (sq7) et de son grand-père. Ce qui s'oppose au monde de la noce chrétienne où la sexualité semble toute sublimée (sq3) ou à celui des religieuses qui " (avaient) peur de montrer leurs jambes " (dixit le grand-père, sq5) mais dont la pratique plus ou moins sadique (mettre un enfant sur le grill) évoque beaucoup de refoulé. 
Enfin, ce thème de la sexualité est intimement lié à un troisième, celui de la religion. Si Giulietta reste marquée par celle de son enfance, les autres personnages, libérés sexuellement, s'avèrent tout aussi hantés par diverses figures du religieux (spiritisme, sq1; religion orientale, sq3; ou de l'antiquité, sq7). Plusieurs d'ailleurs éprouvent, plus ou moins, le besoin de fonder ou d'exprimer leur sexualité dans un langage religieux. Susie, l'affranchie, se fait rassurer sur sa propre bonté par un franciscain plus ou moins intelligent et simoniaque ( $\mathrm{sq} 5$ ). Lors de son party, on évoque les rituels érotiques de l'antiquité égyptienne (sq7). Dans un premier temps, Giulietta a été fascinée par le discours kamasutrien de Bhisma (sq3). Dolorès justifie son ardeur sexuelle par l'identification de Dieu au corps mâle (sq1 et sq4). Les plus rationalistes, les laïcisants, recourent au langage religieux pour exprimer la force de l'éros. Lors de l'arrivée solennelle de Susie sur la plage, le docteur se dit prêt à croire à ce "genre d'apparition» (sq2) et le grand-père avoue éprouver "un sentiment religieux » devant la belle trapéziste (sq4).

\section{Une première lecture : une histoire de cas}

Essayons maintenant de donner un sens à l'ensemble de ce film à partir de ces différents axes. Comme le récit de Giulietta degli spiriti se présente à la manière de l'Ulysse de James Joyce et se déroule largement au fil de la conscience de son héroöne, on peut y lire une histoire de cas psychologique. Giulietta, une femme près de la quarantaine et qui semble avoir tout pour être heureuse, se trouve en pleine crise. Son mari la trompe. Elle revit des états de conscience de son enfance où elle était visitée par des esprits. Enfin, elle est tentée de suivre l'exemple de Susie, sa voisine aux mours libres. Cette crise manifeste une psychologie particulière.

Giulietta est insécure. Dès la première scène ( $\mathrm{sq} 1)$, elle multiplie les essais de perruques pour séduire son mari, lors du repas de leur anniversaire. Elle accepte, sans mot dire, l'oubli de Giorgo d'un événement aussi important et entreprend de servir ses amis non annoncés. La déclaration d'Olaf, lors de la séance de spiritisme - «Elle n'est rien! Elle n'est personne! Elle ne compte pour personne! " (sq1) -, traduit bien son manque d'estime de soi. Cet état dépressif peut s'expliquer par le milieu familial et religieux où elle a été formée. Sa mère, femme d'une grande beauté, mais personnage froid (elle refuse le baiser de Giulietta, sq2) et très stricte (elle n'a pas pardonné la fugue de son père, sq4) s'avère pour elle un modèle écrasant. Notons son manque de sympathie alors que Giulietta la supplie de l'aider (sq9). La sévérité du monde des religieuses asexuées et dépersonnalisées qui l'ont éduquée n'a pu que renforcer son 
mépris d'elle-même. D'ailleurs, le salut qu'elles proposent passe par une mort affreuse.

Au-delà de sa thématique existentielle, cette genèse permet de mieux comprendre la manière dont Giulietta perçoit les gens qui l'entourent. Elle a du mal à se situer de façon réaliste ou, pour le moins, symétrique avec ses pairs. Elle a vite fait, la plupart du temps, de les idéaliser ou encore, plus rarement, de les mépriser. Ainsi, elle voit ses sœurs et sa mère comme des paragons d'une élégance ou d'une excentricité qui lui semblent inatteignables. Si elle a d'abord été fascinée par Bhisma ou le bel Indien, elle a vite fait de les réduire à des figures plus ou moins sordides.

Lorsque sa vision, toute idéale et fusionnelle du mariage ( Il était tout pour elle et elle était toute pour lui ", sq4), s'avère remise en question, elle est renvoyée à l'alternative classique: être vierge-martyre ou putain. Dans la perspective des instances freudiennes de la personnalité, on peut dire que l'énorme surmoi de Giulietta écrase aussi bien son "ça" que son "je". Sa crise s'exprime alors dans l'ambivalence entre la soumission au "surmoi " ou au "ça" : s'identifier à la sainte, à Laura, la suicidée, ou à sa bonne, Elysabeth, ou encore, obéir à Mâ̂tre Susie (sq 5 et sq7).

Pourtant, au fond de sa mémoire, Giulietta garde la figure d'un grandpère qui a su résister aux forces surmoïques (le directeur et l'évêque avec ses menaces de l'enfer) et intégrer son érotisme (dans une fugue qui paraît à Giulietta, tout compte fait, sympathique). Son grand-père l'a délivrée d'une scène sadique où elle se trouvait, encore enfant, à jouer le rôle d'une martyre brûlée vive. Giulietta trouve dans le souvenir de son grand-père - que déjà, toute jeune, elle allait retrouver, en cachette, malgré l'interdit maternel - la force d'envisager d'assumer sa sexualité 6 et sa vie indépendamment de Giorgio son mari manipulateur, insensible et infidèle. Elle se prendra timidement en main, en fréquentant des lieux interdits par son éducation (séance de spiritisme, sq1; consultation d'un guru, sq3; fréquentation de femmes aux idées peu orthodoxes: Dolorès, sq6, et la psychanalyste, sq8) ou des gens aux mœurs répréhensibles

6 Ce que l'on sait de Giulietta laisse facilement supposer une sexualité fort réduite qui pourrait expliquer en partie (sans nécessairement justifier) les écarts de Giorgio. On peut alors lire l'audience avec l'hermaphrodite Bhisma, qui a des connotations fortement onaniques, comme le moment de l'assomption par Juliette de son autoérotisme et sa rencontre avec l'éphèbe comme celle de son autonomie dans le choix de ses partenaires. Après cette rencontre et malgré sa crise de culpabilité, elle ira se serrer contre l'hidalgo et écoutera l'ouverture de Césarino. 
(Susie et sa cour, sq7). Ses contacts avec l'hidalgo inaccessible et le bel Indien, la jetteront dans un désarroi qui atteindra son paroxisme au moment de son affranchissement de Giorgio. Assaillie, plus que jamais, par toutes ses figures surmoïques, ces modèles de vie qu'elle trouvait dans son entourage, elle en vient à douter de l'existence même de ceux qui l'entourent (sq9). Elle revivra alors cette scène, fondamentale pour elle, la séance scolaire et, cette fois, elle résistera aux imprécations maternelles et ce sera, elle-même, la Giulietta-de-quarante ans qui délivrera la Giulietta-enfant de ses liens au grill mortifère.

Les esprits s'estomperont. Giulietta éprouvera une certaine mélancolie de voir partir son grand-père avec la belle Susie. Celui-ci lui révèlera alors la nature de ses esprits. "Nous ne sommes que tes pensées ( «intentioni », en italien), mais toi, tu es la vie». Giulietta comprendra alors ce qu'un thérapeute pourrait nommer explicitement, c'est-à-dire que ses "esprits" n'étaient à la fois que des expressions imaginaires de sa difficulté de vivre (sa névrose) et des moyens de l'assumer et de la résoudre. Libérée de ses projections (guérie?), elle pourra respirer librement et jouir de sa liberté conquise (sq10). Mais voilà que tout recommence et qu'elle se trouve, à nouveau, sollicitée par la prière de nouveaux esprits amicaux. La nature des esprits de Giulietta ne s'épuise donc pas dans l'expression névrotique d'une sexualité ou d'une personnalité mal assumées? L'histoire de Giulietra ne se réduirait pas à celle de la résolution d'une névrose. Il faut donc reprendre la lecture pour rendre compte de tous ses éléments.

\section{Une deuxième lecture : un voyage initiatique}

En fait, cette œuvre fellinienne est beaucoup plus qu'un film psychologique. C'est aussi une fresque de mœurs de la société des années 60 . On y trouve aussi bien la dramatique existentielle caractéristique de l'époque que l'éventail des différentes solutions qui s'y offraient. Giulietta n'est pas seule à vivre une crise identitaire. La plupart des personnages sont au prise avec ce genre de problème : de Giorgio qui doit choisir entre deux femmes aux personnages doubles que sont Bhisma (hermaphrodite, il a, par nature, une double identité), le Maestro efféminé, le vieux Césarino (qui soupire auprès de Giulietta mais remarque les jeunes culturistes au gymnase) et, enfin, le détective (par son métier, mais aussi par sa personnalité, il passe, à plusieurs sens du terme, du rôle d'espion à celui de psychologue ou de confesseur). Les femmes vivent cette crise d'identité de façon plus aigüe. Valentina et Susie (qui oscille entre la femme du monde et la tenancière) envient la vie quiète et heureuse de Giulietta. Même les personnalités qui semblent les plus solides et les mieux intégrées, le grand-père et le médecin gardent quelque chose de schizoïde. Ces deux 
rationalistes ne peuvent exprimer la force de la beauté de Susie qu'en lui conférant un caractère religieux. Cette société fellinienne, pour affranchie qu'elle soit de la chrétienté, reste hantée par le religieux, quand elle n'est pas en quête de salut. En témoignent les nombreuses références aux religions anciennes ou hétérodoxes ou encore la prière de Valentina, à l'esprit Iris : «Dis-nous quelque chose qui nous fera du bien à tous! ».

En même temps, ce film présente toute une série de modèles de valeurs susceptibles de résoudre ce genre de crise identitaire, éthique ou religieuse: figures de survie du christianisme (la scène des noces, sq5; références augustiniennes, sq4 et sq8); figures religieuses alternatives (spiritisme, sq1; hindouisme, sq3; antiquité, sq7); idéaux de féminité (de la femme consommatrice élégante, sql et $\mathrm{sq} 2$; à la femme au foyer, sq3, en passant par les figures du sex symbol, sq2, sq5 et sq7, et de la mystique martyre, sq3) ou encore figures d'idéaux féministes ${ }^{7}$ (la théologie du corps de Dolorès, sq4; le sens de l'autonomie de Gabriella, sq8); figure disciplinaire (le directeur, sq4) et, enfin, figures de psychologie humaniste (classicisme de l'hidalgo, sq3 et sq8; ou jungisme de la psychanalyste avec ses références archétypales, sq8 8$)^{8}$.

On peut voir le personnage de Giulietta comme étant la métaphore de tous ces personnages en crise d'identité, et lire sa démarche de protagoniste comme celle du héros d'un voyage initiatique ${ }^{9}$. Ce voyage se fait sur place, dans le labyrinthe constitué par la mosaique des valeurs contradictoires qui la sollicitent. Son épreuve initiatique consiste à se redonner une identité au travers des modèles qui manifestent ces valeurs. $\mathrm{Ce}$ faisant, elle révèle que toute initiation s'avère travail, sinon procès herméneutique.

L'identité première de Giulietta (façonnée par le conformisme de sa mère et des religieuses qui la réduisaient au modèle de femme au foyer) se

7 S'il s'est souvent présenté comme un Casanova (il a d'ailleurs consacré un film au personnage), Fellini est sans cesse fasciné par le féminisme auquel il consacrera d'ailleurs un film, City of Women (Italie, 1980).

8 Notons qu'il n'y a pas de figure proprement politique (c'est-à-dire qui propose une libération collective) dans le monde de Giulietta.

9 On retrouve l'archétype du voyage initiatique dans la plupart des films felliniens de La Strada (Italie, 1954) à Amarcord (Italie, 1973) en passant par Le notte di Cabiria (Italie, 1957) et Fellini Satyricon (Italie, 1969). Notons que le voyage ne se fait pas toujours dans un espace externe à la vie quotidienne (ex: Amarcord) et que souvent, il s'agit en quelque sorte d'une deuxième initiation c'est-à-dire d'une reprise de la première qui n'aurait pas réussi pleinement. Par exemple, Lo Sceicco Biancho (Italie, 1952). 
trouve remise en cause par l'indifférence et l'infidélité de Giorgio. Celuici qui devait, dans la logique de Giulietta, être "tout pour elle", manifeste sa limite. Le cocon est brisé, c'est la fin du monde, le chaos éliadien, l'enfer. Giulietta se retrouve, vulnérable, comme un enfant de six ans, livrée à ses cauchemars où apparaissent une série de figures auxquelles elle pourrait s'accrocher. Elle perçoit, cependant, tour à tour, le caractère ambigü (fascinant et limité) de chacun de ces cas de figures. Dès la première scène, elle renonce au consommérisme élégant (à sa série de perruques) et choisit de se présenter avec sa propre coiffure. La froideur de Giorgio, son infidélité qu'elle pressent de plus en plus, le retour de ses esprits l'amènent à se rendre chez Bhisma (sq3). Le dîner de noces, bien catholique, qu'elle entrevoit en route, lui apparaît comme un souvenir pieux, sans plus. Elle a vite perçé les élucubrations des disciples de Bhisma, et si elle s'avère fascinée par le caractère d'autoérotisme de la transe de Bhisma, elle se rebiffe lorsqu'il parle de l'amour comme d'un métier. Malgré un tempérament victimaire et les discours conciliateurs du détective, elle trouvera dans l'image du Directeur, la force de résoudre sa crise par le recours à une procédure de divorce (sq4). Cette perpective la renvoie à la solitude. Sous l'influence de Dolorès, dont elle refuse pourtant la théologie érotique, elle sera tentée de basculer dans le monde permissif de Susie. La beauté de celle-ci, l'ivresse, la force presque sacrée de la sexualité qui sature son monde, révèlent aussi à Giulietta les limites de ce monde (sq5 et sq7). La maison de Susie qui s'affiche comme un château de volupté ${ }^{10}$ prend souvent des allures de lupanard ou d'aile psychyatrique en climat de crise. Giulietta ne trouvera pas le salut dans les bras d'un éphèbe. Après avoir franchi toute une série d'interdits, elle se retrouve bourrelée de culpabilité, plus seule que jamais, presqu'incapable de croire à la réalité des personnes concrètes qui l'entourent.

Malgré tout, elle se blottira dans les bras de l'hidalgo (sq8) dont le discours humaniste plutôt abstrait (proverbe espagnol, référence augustienne) s'accompagne d'un souhait d'une sincérité dont elle ne peut douter. Alors qu'elle semble avoir évité l'exhibitionnisme vulgaire du jeu du psycho-drame, elle acceptera le discours démystifiant de la psychanalyste ("Vous dites que vous avez peur d'être abandonnée, ... au fond, vous voulez de tout coeur que votre mari parte... mais vous avez peur d'être heureuse. ») et son évocation de l'archétype des “ arbres qui croissent par eux-mêmes, plongent leurs racines dans la terre et s'élancent vers le ciel " (sq8).

10 Elle fait penser à cette fameuse maison de Chicago où Hugh Hefner, le directeur de Playboy, vivait selon l'idéologie de cette revue. Cette maison faisait rêver bien des gens « libérés » de l'époque. 
Giulietta trouve chez l'hidalgo et la psychanalyste deux mentors qui lui permettent de se ressaisir comme sujet autonome. Elle sera capable d'affronter les minautores. D'abord, Gabriella, sa rivale, sa Mort, qui évite le combat ( Je n'aime pas faire souffrir les gens", dit-elle, en refusant de rencontrer Giulietta) et fait preuve d'une autonomie et d'une lucidité toute féministe que Giulietta intégrera. Elle laissera Giorgio partir sans récrimination et pourra, enfin, affronter la terreur de sa scène primitive que l'on pourrait aussi appeller son mythe fondateur (sq10).

Désespérée du départ de Giorgio, hantée par tous ces surmois, rejetée par sa mère qu'elle implore, Giulietta retrouvera ses racines. Elle parviendra à entendre ses pleurs de jeune enfant qui répugnait à vivre le martyre mais qui avait accepté, sans rien dire (comme disait le grand-père), le discours morbide des religieuses et le conformisme de sa mère. Aux injonctions imprécatoires de cette dernière, ("Obéis à ta mère. Ne bouge pas! Tu ne dois pas! ») Giulietta rétorque: "Tu ne me fais plus peur! ». Le mythe fondateur et toutes ses reformulations s'abolissent d'eux-mêmes. Giulietta n'est pas éternellement condamnée à obéir à des voix surmoïques. Elle peut exister par elle-même, décider de son écoute.

Or, ce voyage initiatique de Giulietta n'est pas qu'affranchissement d'un mythe mortifère. C'est, en même temps, son apprentissage à vivre avec sa mythologie. Si l'on admet avec Eliade que la dynamique profane/sacré persiste dans la conscience sécularisée, il est possible de réaliser que tout au long de son parcours Giulietta s'est livrée à cette dialectique de façon de plus en plus consciente. La Giulietta qui se sentait insignifiante, "en manque d'être", pourrait-on dire pour paraphraser la définition éliadienne du profane, s'est livrée à des hiérophanies, à l'apparition de personnages qui lui semblaient chargés d'être ("sacrés ", au sens éliadien). À chaque fois, elle a été fascinée et troublée.

$\grave{A}$ chaque fois, elle les a mises en procès, rejetant ce qui lui apparaissait loufoque et en gardant ce qui pouvait la nourrir. L'hidalgo et la psychanalyste n'ont pas été ses seuls ni ses premiers guides. Valentina l'a sortie de sa torpeur domestique (sq1 et sq3), le Maestro a reconnu sa capacité d'entrer en contact avec des esprits (sq1), ce qui l'a aidée à résister au rationalisme du médecin et aux patenôtres de l'évêque (sq3). Bhisma a réveillé un érotisme qui s'était sans doute perdu sous une sexualité d'habitude, Susie lui a révélé la possibilité de se retrouver désirable et désirante (sq4 et sq7) et Gabriella, au nom d'ange, lui a rendu la lucide autonomie que revendique le féminisme (sq9). Loin de pratiquer une "religion à la carte", de se livrer confortablement à un choix aléatoire devant diverses possibilités d'être au monde (sq5 et sq7), Giulietta s'est battue avec chacune de ses «hiérophanies » pour en tirer la substance nécessaire à la reconstruction de son identité. C'est après ce long travail avec ces mul- 
tiples guides qu'elle a pu entendre l'hidalgo et la psychanalyste et affronter un combat dont la résolution ultime dépasse sa victoire sur sa mère.

$\mathrm{Si}$ les esprits se sont estompés à ce moment, ils ne sont pas tous disparus. Giulietta ne voulait pas renoncer à son grand-père, elle voulait qu'il l'amène avec lui. Celui-ci, qui l'avait délivrée une première fois de la scène du martyre et qui a été son premier guide, lui permet alors de faire une dernière prise de conscience. Ces esprits qui ont hanté Giulietta n'ont pas de substance par eux-mêmes. C'est elle, par sa vitalité, qui les a fait vivre. C'est elle qui les a générés au fil de ses rencontres pour dire son mal de vivre, mais aussi laborieusement pour s'en libérer. Les esprits ne sont pas que méchants, ils peuvent être bienveillants. S'ils aliènent, ils peuvent aussi aider à se libérer. Ils ne sont pas hors de nous, ils sont nôtres, de notre vie, une médiation que nous trouvons pour nous aider à vivre. Libérée de son mythe fondateur mortifère, de son imaginaire névrotique, interpellée par d'autres de ces créatures, tout aussi imaginaires, Giulietta découvre ce régistre d'elle-même qu'elle peut décider d'assumer. L'expérience de la libération d'un mythe n'implique pas nécessairement l'affranchissement de la mythologie. Cela peut se révéler l'occasion d'apprivoiser sa capacité mythique. Giulietta échappe au sort du "taureau (qui) meurt dans sa propre chimère ", elle a trouvé "la douceur des gestes, l'équilibre,... la pureté de la pensée ", qui lui permettront de vivre avec ses esprits.

\section{Les figures du diable dans Giulietta degli spiriti}

On peut donc se demander quelle est la figure du diable dans le monde de Giulietra et plus particulièrement pour elle-même. Une première réponse, négative. La figure du diable qui hante Giulietta n'est pas celle du monde de la chrétienté (de l'Exorcisme, par exemple) c'est-à-dire une personne réelle qui s'oppose à Dieu et qui peut posséder les humains et les entraîner dans l'enfer éternel. Dans le film, il n'y a que quelques personnages secondaires qui véhiculent une telle image. Giulietta, pour sa part, n'a pas cru l'évêque qui prétendait que son grand-père avait pactisé avec le diable ni aux menaces du directeur qui le vouait à l'enfer (sq4). Même si elle garde des traces importantes de son éducation chrétienne, Giulietta vit dans un monde de post-chrétienté, un monde moderne, sécularisé, rationaliste, qui ne croit « ni à Dieu, ni a diable " et où l'Église, par ses attitudes idéalistes, angéliques et perverses, a perdu pertinence et crédibilité.

Une deuxième réponse. En même temps, Giulietta résiste à cette modernité qui, comme le fait son ami médecin ( $\mathrm{sq2}$ ), réduit, par une espèce de freudisme primaire, l'imaginaire à une mauvaise intégration de la sexualité. En ce sens, on pourrait dire que son personnage s'inscrit 
dans la post-modernité. Le fait de ne pas croire à l'existence du diable n'épuise pas, pour elle, la question de la réalité du diable, ou encore d'esprits qui peuvent hanter ou posséder des personnes. Elle expérimente concrètement dans sa société comme dans son propre vécu la présence, bien concrète, de forces malignes voire mortifères ${ }^{11}$.

Le monde, la société que Giulietta appréhende et où elle saisit sa propre existence ne tiennent pas leurs promesses de rationalité, de liberté, voire d'hédonisme qui caractérisent souvent la modernité. On pourrait d'ailleurs penser que son enracinement chrétien et bourgeois l'ont aidée à en saisir assez rapidement les ambiguités. Quoiqu'il en soit, notre deuxième lecture a montré comment Giulietta répugnait, malgré ses fascinations (on pourrait presque dire "ses tentations"), à s'identifier aux différents modèles, aux différentes " hiérophanies " qui se manifestaient à elle. Malgré sa façade optimiste, le monde de Giulietta est marqué par le mal, sinon par le Malin. Notons que cette marque est universelle. Rien n'y échappe. L'Église elle-même y apparaît décrochée du réel (scène idéalisée du mariage), voire perverse (religeuses asexuées et sadiques). Cela peut scandaliser les tenants de la chrétienté qui ont oublié que le Jésus des évangiles a traité de Satan $(M c 8,33)$ celui-là même auquel il a confié les clés du Royaume. Implicitement, on pourrait retrouver dans ce monde, en y incluant l'Église, l'archétype du péché originel.

Il n'est alors pas surprenant qu'une personnalité fragile et en crise, comme celle de Giulietta, perçoive ces réalités pour le moins ambiguës sous formes d'esprits troublants et menaçants. Mais si ces esprits, qui la hantent et la torturent, peuvent rappeller les présences démoniaques des cas de possession, ils ne s'y identifient pas complètement. Ils ne sont pas l'œuvre mauvaise d'un démon extérieur à Giulietta qu'il s'agirait de chasser de sa vie par un exorcisme quelconque, avec ses formules canoniques de prière et de jeûne. Il s'agit, plutôt de présences endogènes et ambivalentes dont Giulietta parviendra, en les assumant courageusement, à soupçonner leur nécessité, leur sens et leur fonction. Ces esprits se sont

11 Cette position de Giulietta se rapproche de celle du médecin dans Rosemary's baby (POLANSKI, Roman POLANSKI, ÉUA, 1968), un film de la même époque où Satan joue un rôle important. La protagoniste, Rosemary, découvre que l'enfant, dont elle est enceinte, est considéré par une secte comme l'incarnation de Satan. Elle demande à un médecin, dont elle implore l'aide, s'il croit à l'existence de Satan. " Je n'y crois pas ", dit-il, "mais je crois que dans une ville comme New York, il peut y avoir des gens assez malades pour y croire ". Son refus de croire en l'existence de Satan, n'empêche pas ce médecin de reconnaître qu'en termes psycho-sociaux (une ville comme New York) ou individuels (certaines personnes), Satan garde concrèment une existence symbolique. 
avérés la seule voie qu'elle a trouvée pour manifester à la fois le mal de vivre qu'elle refoulait et la limite des modèles de vie alternatifs qui l'entouraient. Leur difficile fréquentation lui a permis de discerner entre les dimensions mortifères et nourissantes de ces présences.

Elle a alors pu se rendre compte de la fonction, tout compte fait, positive de ces présences. En fait, ce genre d'esprits a joué, pour elle, un rôle qui se rapproche de celui de "l'avocat du diable", c'est-à-dire de celui qui en relevant les zones d'ombre de la vie d'un candidat permet d'assurer un meilleur discernement dans un procès de canonisation. Dans leur propre registre et avec leur propre logique dialectique, celui de l'imaginaire, les esprits ont permis à Giulietta, cette femme sécularisée, de se donner une intelligence sinon de sa foi religieuse, du moins de ce don, qui n'est pas de l'ordre de l'évidence ou de la rationalité scientifique, que nous nous faisons d'assumer notre vie.

Notons, enfin, que cette vision du diable (ou des esprits), moins comme personne rebelle qui s'oppose à Dieu pour lui ravir les humains (image qui habite la tradition chrétienne), mais comme facteur symbolique d'un discernement, se rapproche peut-être des premières apparitions du diable dans l'Ancien Testament. Qu'on pense, par exemple, au livre de Job. Il s'agit alors d'un ange, qui n'est pas encore déchu mais qui fait partie intégrante de la cour, du conseil de Dieu. Son rôle est justement de tempérer l'enthousiasme de Dieu, de l'aider dans son discernement en lui suggérant d'éprouver ses positions par des scénarios plus sombres mais, sans doute, valables puisque Dieu y consent.

L'image d'un Dieu pour qui tout n'est pas joué dans sa toute puissance et qui serait, lui-même, aux prises ayec un imaginaire, a quelque chose, sinon de fellinien, du moins de fascinant. Tout comme les idées que le diable, malgré l'incroyance qu'il suscite chez les modernes et même chez de nombreux théologiens post-Vatican II, continue d'exercer un pouvoir symbolique réel dans nos sociétés, comme dans des consciences individuelles et qu'il puisse, en l'occurrence, jouer un rôle positif. N'étant ni bibliste, ni théologien fondamentaliste, je me contente de les évoquer. De toute façon, il faudrait les reprendre dans un autre article ou... dans un autre film. Il reste qu'un film comme Giulietta degli spiriti amène à ce genre de questions. 